Letters to the Editor

\title{
Investigation of Copper(II)-Binding Behavior of Fulvic Acids by Three-Dimensional Fluorescence Spectrometry
}

\author{
Masami Fukushima*†, Shunitz Tanaka**, Ken Nakayasu**, Keiko Sasaki***, \\ Hiroshi NAKaMURA** and Kenji TATSUMI* \\ * Department of Hydrospheric Environmental Protection, National Institute for Resources and Environment, \\ Onogawa, Tsukuba 305, Japan \\ **Division of Material Science, Graduate School of Environment and Earth Science, \\ Hokkaido University, Sapporo 060, Japan \\ ***Faculty of Engineering Hokkaido University, Sapporo 060, Japan
}

Keywords Fulvic acid, copper(II) complexation, three-dimensional fluorescence spectrometry, excitation-emission matrix

Fulvic acids (FAs), which widely are distributed in soils and natural water, are complex mixtures of highmolecular-weight organic compounds. In spite of reporting on many common functional groups and structural features ${ }^{1,2}$, their characteristics are different from each other. The complexation of metal ions with FAs has been studied from the point of view of the speciation of metal ions in natural water. ${ }^{3}$ Especially, fluorescence-quenching spectrometry can give various types of information about complexation between FAs and heavy-metal ions, such as $\mathrm{Cu}^{2+}, \mathrm{Mn}^{2+}, \mathrm{Co}^{2+}$ and $\mathrm{Pb}^{2+}{ }^{4-10}$ Fluorescence-quenching spectrometry offers a unique perspective on metal-FA binding, because it can directly observe the binding sites of FA which are related to fluorophores. FAs are complex mixtures containing various fluorophores, and the quenching of FA by metal ions at a certain excitation wavelength represents complexation in a specific binding site to the metal ions. However, fluorescence quenching of FA by complexation with metal ions has been observed at a single excitation wavelength. In order to show various metalbinding sites in FA, Cabaniss studied the synchronous fluorescence-quenching spectra. ${ }^{10}$ He showed that FA caused quenching by complexation with metal ions over a wide range of excitation wavelengths.

On the other hand, three-dimensional fluorescence spectra (3D spectra) provided a complete representation of the fluorescence spectral features of FAs in the form of an excitation-emission matrix (EEM), in which the fluorescence intensity is presented as a function of the excitation wavelength on one axis and the emission wavelength on the other. Recently, Mobed et al. reported on the effects of the FA concentration, ionic

\footnotetext{
† To whom correspondence should be addressed.
}

strength, and pH on the EEMs. ${ }^{11}$ However, there have been no papers on the effect of the metal concentration on the EEM of FA. In the present work we observed the 3D spectra of the FAs, which were extracted from soil, and the effect of the copper(II) concentration on the EEMs was investigated.

\section{Experimental}

The FAs were extracted from Shinshinotsu peat (SFA) and Inogashira soil (IFA). The SFA was purified according to a protocol of the International Humic Substances Society (IHSS) ${ }^{12}$, and the IFA was purchased from Japan Humic Substances Society (in Department of Chemistry, Faculty of Science, Tokyo Metropolitan University, Hachiohji, Tokyo 192-03). ${ }^{13}$ The elemental composition and total acidity are summarized in Table 1. Stock solutions of the FAs $\left(100 \mathrm{mg} \mathrm{l}^{-1}\right)$ were prepared by dissolving the FA into doubly distilled water, and were stocked under dark and cool conditions. Gallic acid (GA) was used as a model compound of FA. A copper(II) stock solution $(0.01 \mathrm{M})$ was prepared by dissolving electrolytic copper into a $0.1 \mathrm{M} \quad \mathrm{HNO}_{3}$ aqueous solution.

Three milliliters of a test solution containing 0.5 or $1 \mathrm{mg} \mathrm{l}^{-1}$ of the FA solution, adjusted at $\mathrm{pH} 4.5$ by $0.1 \mathrm{M}$ acetate buffer, was pipetted into a quartz cell $(1 \mathrm{~cm} \times$ $1 \mathrm{~cm}$ ) with a micro-magnet bar for stirring. After the stock solution of copper(II) was diluted up to 1-5 mM, a $10-50 \mu \mathrm{l}$ aliquot of the copper(II) solution was added to the FA solution with a micro-pipette (Eppendorf). The fluorescence emission spectra were measured at various excitation wavelengths $(220-400 \mathrm{~nm}$ for every $10 \mathrm{~nm})$. The emission wavelength range was from $350 \mathrm{~nm}$ to 
Table 1 Elemental composition and total acidity of the FAs

\begin{tabular}{|c|c|c|c|c|c|c|c|}
\hline FA & Origin & $\% \mathrm{C}$ & $\% \mathrm{H}$ & $\% \mathrm{~N}$ & $\% \mathrm{~S}$ & $\% \mathrm{O}$ & Total acidity/meq $\mathbf{g}^{-1}$ \\
\hline SFA & Shinshinotsu peat & 41.26 & 5.27 & 0.95 & 0.36 & 52.16 & 10.2 \\
\hline IFA $^{a}$ & Inogashira soil & 43.28 & 3.47 & 1.73 & 0.08 & 51.44 & 10.9 \\
\hline
\end{tabular}

a. The values of IFA are certified values in the literature. ${ }^{13}$
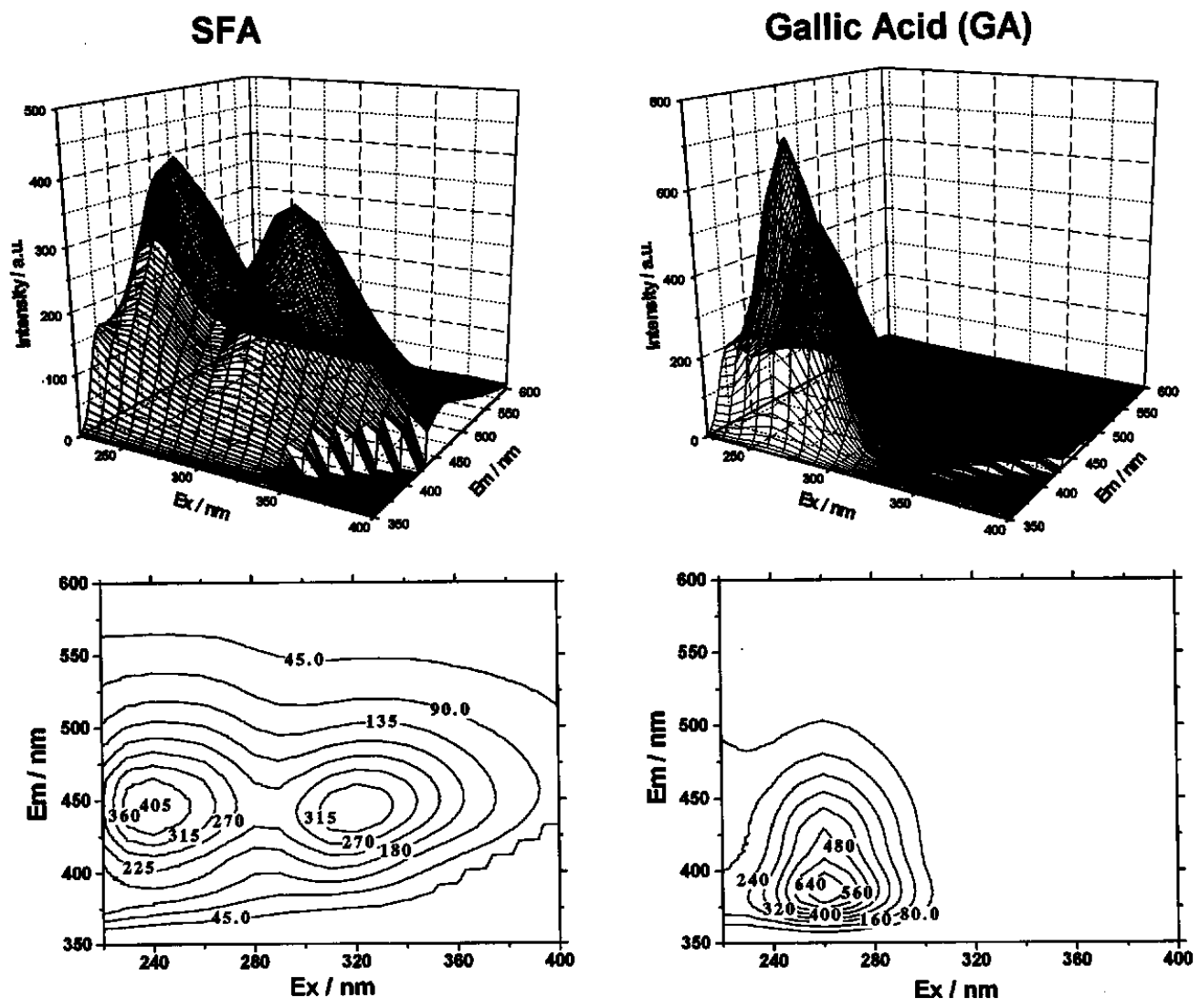

Fig. 1 Three-dimensional fluorescence spectra of SFA and the model compound (GA). SFA, $1 \mathrm{mg} \mathrm{l}^{-1}$; GA, $0.5 \mathrm{mg} \mathrm{l}^{-1} ; \mathrm{pH} 4.5$ by $0.1 \mathrm{M}$ acetate buffer, $25^{\circ} \mathrm{C}$.

$600 \mathrm{~nm}$, and the wavelength step size was $1 \mathrm{~nm}$. A Shimadzu RF-5300 PC type spectrofluorometer was used, and the excitation/emission slits on the monochromator were set to $20 \mathrm{~nm} / 15 \mathrm{~nm}$. The temperature of the cell was maintained at $25^{\circ} \mathrm{C}$. The emission spectra of a blank solution without FA were measured at every excitation wavelength, and were subtracted from the spectra for the FAs. The data set at each excitation wavelength were converted into 3D spectra and EEMs using computer graphic software, MicroCal Origin Ver. 3.01 .

\section{Results and Discussion}

\section{$3 D$ spectrum and EEM for the $F A$}

Figure 1 shows the $3 \mathrm{D}$ spectra as well as the excitation and emission matrices (EEMs). The 3D spectrum of SFA is clearly different from that of a simple compound, GA. Although the 3D spectrum of GA showed one sharp peak (EX/EM: $260 / 387 \mathrm{~nm}$ ), two broad peaks $(240 / 445 \mathrm{~nm}$ and $320 / 444 \mathrm{~nm})$ appeared in the SFA. In the case of IFA, two broad peaks were also observed $(250 / 444 \mathrm{~nm}$ and $320 / 441 \mathrm{~nm})$. Such differences between the FAs and GA showed that the FAs were complex mixtures containing various types of fluorophores. Two fluorescence peaks in the 3D spectra suggest that there are two types of fluorophores in the FAs. One might have been phenolic compounds, such as GA, which was identified in soil FA by Schnitzer et al. ${ }^{14}$ Another type of fluorophores might be regarded as being anthraquinone dyes, because this existence in the structure of the humic substances was reported by Cheshire et al. ${ }^{15,16}$ Moreover, Mobed et al. showed that 
SFA: $1 \mathrm{mg} \mathrm{L}^{-1}$
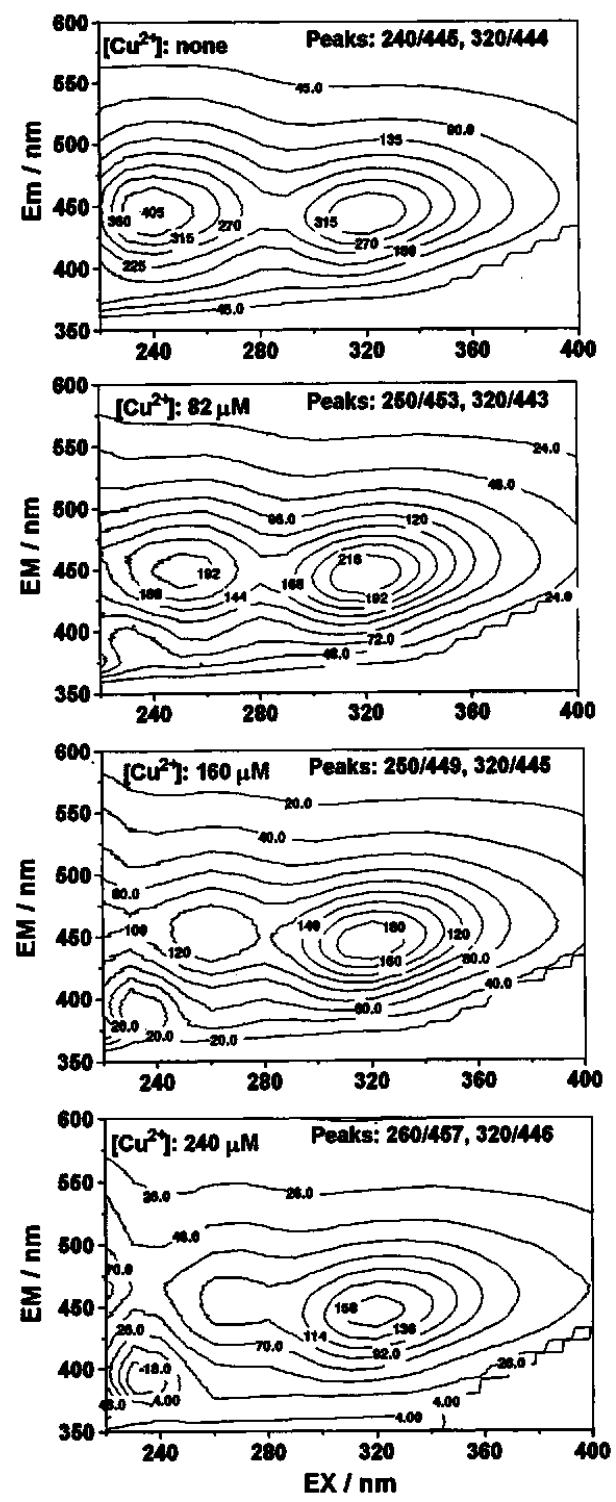

IFA: $0.5 \mathrm{mg} \mathrm{L}^{-1}$
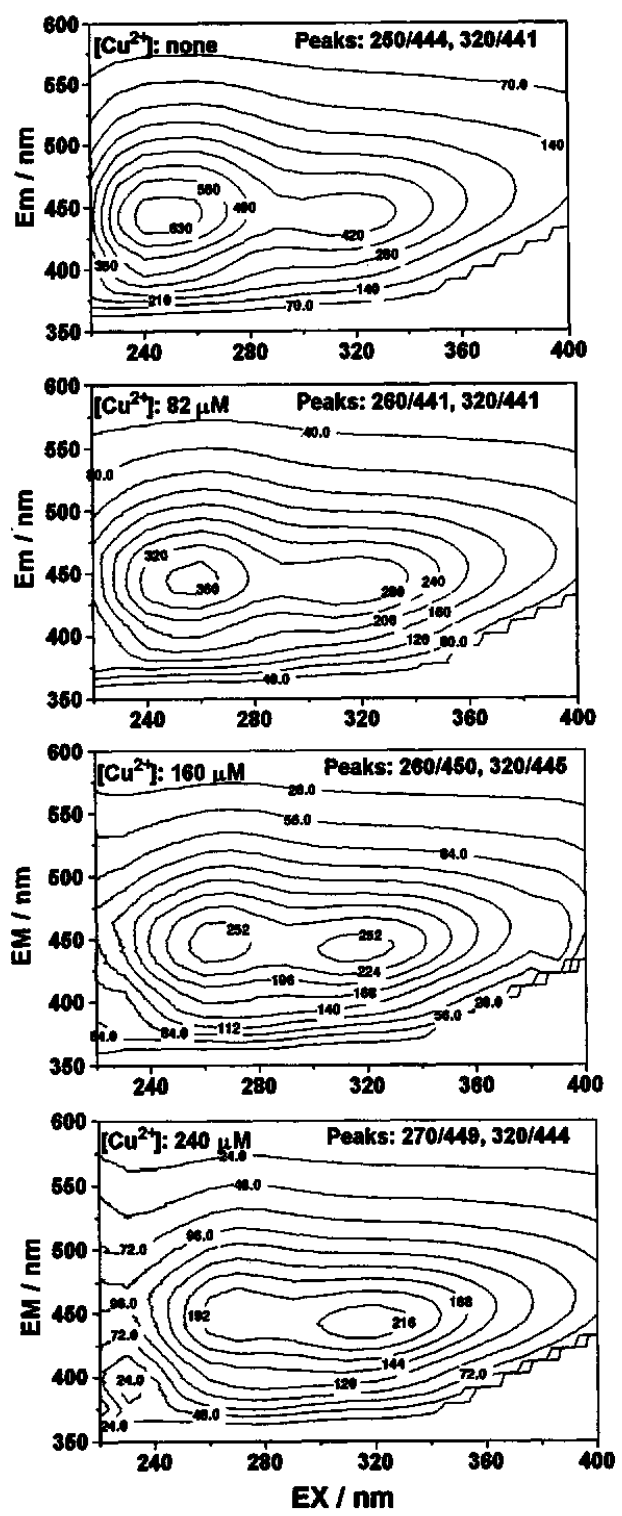

Fig. 2 Effect of the copper(II) concentration on the EEMs of SFA and IFA.

various IHSS humic substance reference materials had two EEMs, and the peaks for the EEMs were observed in the range of $300-400 / 400-500 \mathrm{~nm} .^{11}$ The peaks of the EEMs for SFA and IFA in the present work were in the range of $240-320 / 440-445 \mathrm{~nm}$, and the differences in the emission wavelength between two peaks were small. Such a behavior resembles that for the peat humic acid and the soil humic acid references of IHSS (Fig. 2 and Fig. 5 in ref. 11).

Figure 2 shows the effect of the copper(II) concentration on the EEMs of SFA and IFA. The EEMs of the FAs had two peaks, in which the first and second peaks appeared at 240-270/440-450 nm and 320/440$445 \mathrm{~nm}$, respectively. The fluorescence intensities of each FA decreased along with an increase of the copper(II) concentration; this fluorescence quenching was due to complexation with copper(II) ions. The excitation wavelengths of the first peaks were shifted to a higher wavelength along with an increase in the copper(II) concentrations: SFA, 240 to $260 \mathrm{~nm}$; IFA, 250 to $270 \mathrm{~nm}$. This suggests that the degree of fluorescence quenching at the first peak ( 240 or $250 \mathrm{~nm}$ ) was larger than that in the higher wavelength region. On the other hand, the excitation wavelength of the second peak $(320 \mathrm{~nm})$ did not change in spite of a decrease in the fluorescence intensity with the copper(II) concentration. Such differences in quenching between the first and second peaks might be able to explain the differences in the copper(II) binding abilities at each peak.

Evaluation of copper(II)-binding abilities at two peaks of EEMs To evaluate the copper(II) binding abilities at two 

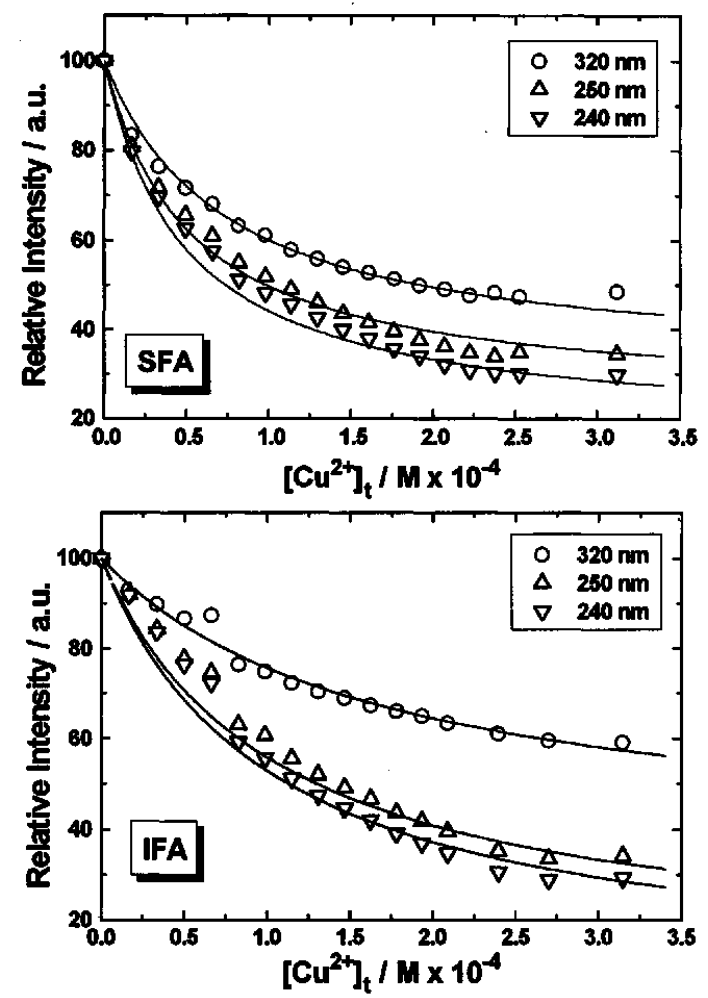

Fig. 3 Relationships between the fluorescence intensity and the copper(II) concentration. SFA, $1 \mathrm{mg} \mathrm{l}^{-1}$; IFA, $0.5 \mathrm{mg}$ $1^{-1}$; $\mathrm{pH} 4.5$ by $0.1 \mathrm{M}$ acetate buffer, $25^{\circ} \mathrm{C}$.

peaks of the EEM, the emission peak intensities at $445 \mathrm{~nm}$ were measured for various copper(II) concentrations at excitation wavelengths of 240,250 and $320 \mathrm{~nm}$, respectively. Figure 3 shows the relationships between the fluorescence intensity and the copper(II) concentration at various excitation wavelength. The observed fluorescence intensity at any point in the titration $(I)$ decreased along with an increase in the copper(II) concentration, hyperbolically.

In the case of a $0.1 \mathrm{M}$ acetate buffer at $\mathrm{pH} 4.5$, the concentration of the copper(II) species without FA was calculated from the stability constant of copper(II)acetate $\left(K_{\mathrm{CuOAC}}=10^{1.8}\right),{ }^{17}$ it was then found that more than 90\% of the copper(II) ions were presented as copper(II) acetate. Assuming a 1:1 complexation between $\mathrm{Cu}^{2+}$ plexation between $\mathrm{Cu}^{2+}$ and $\mathrm{L}_{i}\left(K^{\prime}\right)$. Therefore, $K^{\prime}$ can be defined as the conditional stability constant containing the copper(II)-acetate complexation. Moreover, $K^{\prime}$ can be represented as the stability function used to describe the binding metal ion to $n$ ligands in a multiligand mixture, as described by Ryan et al..$^{5}$,

$$
K^{\prime}=\frac{\sum_{i=1}^{n}\left[\mathrm{CuL}_{i}\right]}{\left[\mathrm{Cu}^{2+}\right] \sum_{i=1}^{n}\left[\mathrm{~L}_{i}\right]}
$$

The mass-balances for the total copper(II) concentration $\left(\left[\mathrm{Cu}^{2+}\right]_{\mathrm{t}}\right)$ and the total ligand concentration $\left(C_{\mathrm{L}}\right)$ are represented as follows:

$$
\begin{aligned}
& {\left[\mathrm{Cu}^{2+}\right]_{\mathrm{t}}=\left[\mathrm{Cu}^{2+}\right]+\sum_{i=1}^{n}\left[\mathrm{CuL}_{i}\right],} \\
& C_{\mathrm{L}}=\sum_{i=1}^{n}\left(\left[\mathrm{~L}_{i}\right]+\left[\mathrm{CuL}_{i}\right]\right)
\end{aligned}
$$

Combining Eqs. (2)-(4), the following relationship between $\left[\mathrm{Cu}^{2+}\right]_{\mathrm{t}}$ and $\Sigma\left[\mathrm{CuL}_{i}\right]$ is derived:

$$
\sum_{i=1}^{n}\left[\mathrm{CuL}_{i}\right]=\frac{C_{\mathrm{L}} K^{\prime}\left(\left[\mathrm{Cu}^{2+}\right]_{\mathrm{t}}-\sum_{i=1}^{n}\left[\mathrm{CuL}_{i}\right]\right)}{1+K^{\prime}\left(\left[\mathrm{Cu}^{2+}\right]_{t}-\sum_{i=1}^{n}\left[\mathrm{CuL}_{i}\right]\right)}
$$

Sarr and Weber showed that the decrease in the fluorescence intensity correlated with the total concentration of the metal-FA complex. ${ }^{4}$ From this concept, they and Ryan derived a relationship between the total concentration of metal-FA complex and the fluorescence intensity, as written below:4,5

$$
\frac{\sum_{i=1}^{n}\left[\mathrm{CuL}_{i}\right]}{C_{\mathrm{L}}}=\frac{I_{\mathrm{L}}-I}{I_{\mathrm{L}}-I_{\mathrm{CuL}}}
$$

where $I_{\mathrm{L}}$ and $I_{\mathrm{CuL}}$ denote the fluorescence intensity at the start of titration and the fluorescence intensity when all of the ligand is bound as the copper(II) complex. Assuming that $I_{\mathrm{L}}=100$, the following equation is derived by combining Eqs. (5) and (6):

$$
I=\left(I_{\mathrm{CuL}}-100\right) \times \frac{\left(K^{\prime}\left[\mathrm{Cu}^{2+}\right]_{\mathrm{t}}+C_{\mathrm{L}} K^{\prime}+1\right)-\sqrt{\left(K^{\prime}\left[\mathrm{Cu}^{2+}\right]_{\mathrm{t}}+C_{\mathrm{L}} K^{\prime}+1\right)^{2}-4 K^{\prime 2} C_{\mathrm{L}}\left[\mathrm{Cu}^{2+}\right]_{\mathrm{t}}}}{2 K^{\prime} C_{\mathrm{L}}}+100 .
$$

and the binding site of FA $\left(\mathrm{L}_{i}\right)$, as described in the literature ${ }^{4-9}$, the ligand-exchange equilibrium between acetate and $L_{i}$ is written as

$$
\mathrm{CuOAc}+\mathrm{L}_{i} \rightleftharpoons \mathrm{CuL}_{i}+\mathrm{OAc}^{-} .
$$

The product of the equilibrium constant for Eq. (1) and $K_{\text {CuOAc }}$ represents the equilibrium constant for com-
Equation (7) shows the relationships between the fluorescence intensity and the total copper(II) concentration at a given $C_{\mathrm{L}}$. The experimental data set $\left(\left[\mathrm{Cu}^{2+}\right]_{\mathrm{t}}, I\right)$ were interpreted by a non-linear least-square regression analysis, and the $K^{\prime}$ and $I_{\mathrm{CuL}}$ values could be evaluated. The total acidity of each FA given in Table 1 was used for the $C_{\mathrm{L}}$ value as a fixed parameter; also, the molar concentrations were given in Table 2 . 
Table 2 Evaluation of conditional stability constants of FAs at various excitation wavelength

\begin{tabular}{ccccc}
\hline \multirow{2}{*}{ FA } & \multirow{2}{*}{ Parameter } & \multicolumn{3}{c}{ Excitation wavelength/nm } \\
\cline { 3 - 5 } & & 320 & 250 & 240 \\
\hline \multirow{2}{*}{ SFA } & $K^{\prime}$ & $7.4 \times 10^{3}$ & $2.0 \times 10^{4}$ & $2.1 \times 10^{4}$ \\
& $I_{\mathrm{CuL}}$ & 31.7 & 24.0 & 16.9 \\
IFA & $K^{\prime}$ & $5.9 \times 10^{3}$ & $9.8 \times 10^{3}$ & $1.0 \times 10^{4}$ \\
& $I_{\mathrm{CuL}}$ & 34.4 & 10.7 & 6.3 \\
\hline
\end{tabular}

$C_{\mathrm{L}}:$ SFA, $1.02 \times 10^{-5} \mathrm{M}$; IFA, $5.45 \times 10^{-6} \mathrm{M}$.

The solid curves in Fig. 3 were calculated by using Eq. (7), and are in good agreement with the experimental data points. The $K^{\prime}$ and $I_{\mathrm{CuL}}$ values, which were evaluated by curve-fitting, are summarized in Table 2 . In each FA, the $K^{\prime}$ values for the lower excitation wavelength ( 240 or $250 \mathrm{~nm}$ ) were slightly larger than that for higher wavelength $(320 \mathrm{~nm})$. This is due to heterogeneity in the binding site of the FAs. The differences in the $K^{\prime}$ values between $320 \mathrm{~nm}$ and 250 or $240 \mathrm{~nm}$ suggest that the peaks of the EEMs correspond to different types of metal-binding sites.

In the present work, we observed the $3 \mathrm{D}$ spectra of FAs and investigated the effect of the copper(II) concentration on the EEMs. The peaks in the EEM represented the principal fluorophores in FAs; also, these fluorescence quenching by copper(II) ions gave useful information about the complexation of copper(II) ions to the specific binding-sites in FAs.

\section{References}

1. F. J. Stevenson and K. M. Goh, Geochim. Cosmochim. Acta, 35, 471 (1971).

2. N. Senesi, T. M. Miano, M. R. Provenzano and G. Brunetti, Soil Sci., 152, 259 (1991).

3. T. M. Florence, Talanta, 29, 345 (1982).

4. R. A. Saar and J. H. Weber, Anal. Chem., 52, 2095 (1980).

5. D. K. Ryan and J. H. Weber, Environ. Sci. Technol., 16, 866 (1982).

6. D. K. Ryan and J. H. Weber, Anal. Chem., 54, 986 (1982).

7. D. K. Ryan, C. P. Thompson and J. H. Weber, Can. J. Chem., 61, 1505 (1983).

8. S. E. Cabaniss and M. S. Shuman, Anal. Chem., 66, 2418 (1988).

9. J. Dobbs, W. Susetyo, F. E. Knight, M. A. Catsles, L. A. Carreira and L. V. Azarraga, Anal. Chem., 61, 483 (1989).

10. S. E. Cabaniss, Environ. Sci. Technol., 26, 1133 (1992).

11. J. J. Mobed, S. L. Hemmingsen, J. L. Autry and L. B. McGrown, Environ. Sci. Technol., 30, 3061 (1996).

12. International Humic Substances Society, "Outline of Extraction Procedures", 1982.

13. A. Watanabe, K. Itoh, S. Arai and S. Kuwatsuka, Soil Sci. Plant Nutr., 40, 601 (1994).

14. M. Schnitzer, S. U. Khan, "Humic Substances in the Environment", pp. 183-189, Dekker, New York, 1972.

15. M. V. Cheshire, P. A. Cranwell, C. P. Floyd and R. D. Howorth, Tetrahedron, 23, 1669 (1967).

16. M. V. Cheshire, P. A. Cranwell and R. D. Howorth, Tetrahedron, 24, 5155 (1968).

17. M. Yasuda, K. Yamasaki and H. Ohtani, Bull. Chem. Soc. Jpn., 33, 1067 (1960). 